IP Periodica Polytechnica

Transportation Engineering

45(1), pp. 1-7, 2017

DOI: $10.3311 /$ PPtr. 8677

Creative Commons Attribution (i)

RESEARCH ARTICLE

\section{The Analysis Method of Capacity and Delay on Entrance Lane with Road-side Bus Lane}

\author{
$\mathrm{Yi} \mathrm{Cao}^{{ }^{*}}$, Zhongzhen Yang ${ }^{2}$, Zhongyi Zuo ${ }^{1}$
}

Received 14 October 2015; accepted 03 March 2016

\begin{abstract}
In order to reveal the traffic characteristics at signalized intersections with road-side bus lane, the analysis methods of capacity and delay at entrance lane were studied. Selecting a signalized intersection with road-side bus lane as investigation location, several traffic parameters were surveyed. Considering the traffic conflict between right-turn vehicles and through buses in this kind of entrance lane and based on the Gap Acceptance Theory, the calculation model of saturation flow rate, which is a key parameter both in the formulas of capacity and delay at signalized intersection mentioned in HCM2000, was improved. Using the investigation data, the calculation results of the traditional model and improved model were compared and analyzed. The research indicated that, comparing with common entrance lane, the capacity of right-turn lane at the entrance lane with bus lane should be reduced, and the travelling delay of bus lane and its inside adjacent lane should be increased. The degree of these effects is related to bus volume, right-turn volume and headway between buses.
\end{abstract}

\section{Keywords}

traffic flow theory, capacity, delay, bus lane, Gap Acceptance Theory, traffic conflict

\footnotetext{
${ }^{1}$ School of Transportation Engineering, Dalian Jiaotong University, Huanghe Rd. 794 Dalian, China

${ }^{2}$ Transportation Management College, Dalian Maritime University, Linhai Rd.1 Dalian, China

*Corresponding author, e-mail: caoyi820619@aliyun.com
}

\section{Introduction}

With extensive development and implementation of public transport priority in many big cities all over the world, more and more bus lanes are set up at the road segments with several bus lines. In China, the road-side bus lane is one of the most common application types. Obviously, for the intersection entrance with road-side bus lane, the function of lanes is varied. Therefore, the original traffic flows need to distribute again in order to adapt to the new lane function. At the same time, traffic conflicts between right-turn vehicle and through bus may occur, as Lian-gui Lei (2008) and Bin Lu (2011) studied. In view of the reason above, in order to adapt to this new traffic organization and management mode, it is necessary to improve the analysis method of capacity and delay. This work will provide a theoretical basis for capacity and delay analysis at the signalized intersection with road-side bus lane.

At present, both domestic and foreign scholars have researched the traffic operation at signalized intersections with road-side bus lane. Using the extensive movement wave model, Chiabaut Nicolas et al. (2012) studied the capacity and travelling time of intermittent bus lane. The research indicated that, although several advantages of this kind of priority mode exist, however, the capacity of bus lane is reduced and the travelling time of buses is also increased, to some extant. By means of traffic simulation, Arasan V. Thamizh et al. (2010) studied the effect of bus lane setting on the travelling speed and degree of saturation of buses and other vehicles, under the traffic condition of highly heterogeneous. According to the actual case, Ismail, R. et al. (2012) compared and studied the reduction of bus travelling delay before and after a bus lane with specific length. By means of automatic vehicle location and passenger counting, Surprenant- Legault et al. (2011) constructed two statistical models for calculating the travelling time and on-time performance of buses. Based on the models, the effect of the two parameters mentioned above is studied after bus lane setting.

Domestically, by analyzing several kinds of measured saturation headway, Jing Zhao et al. (2008) calculated a heavy vehicles adjustment factor at intersection entrance under the effect of bus fleet. Furthermore, with traffic simulation verification, they 
also proposed the capacity models for private vehicles, buses and lane groups. According to the investigation data, Tian-hong Gu et al. (2013) analyzed the effect of bus lane setting on headway of private vehicles and buses. Aiming at median bus lane in urban expressway, Xu-mei Chen et al. (2014) not only proposed the simulation analysis method of capacity at export and entrance ramp of expressway, but also verified the validity of the method by actual data. On the basis of improving the travelling speed model of heterogeneous vehicles, Tao Wang et al. (2014) constructed a time-utility model for calculating the critical condition of bus lane setting with the objective of minimum total travelling time. Based on the analysis of vehicle arrival characteristic in bus lane, Cui-juan Zhu (2006) distinguished the type of bus queuing delay. She also constructed the delay model for every type of queuing delay.

Although several studies on this topic, in general, have been carried out both here and abroad, most of them focused their attention on capacity and delay analysis of bus lane itself, rather than the effects on them in its adjacent private lane. In addition, the attention on traffic conflicts between right-turn vehicles and through buses after road-side bus lane setting are inadequate. Given this status in the literature, based on the traffic investigation, the characteristics of traffic conflict and headway distribution are considered mainly in this paper. Using the Gap Acceptance Theory, the traditional methods of capacity and delay for signalized intersection mentioned in HCM2000 are also improved. There are significant theoretical and practical applications to be found when calculating capacity and delay for signalized intersections with road-side bus lane.

\section{The traditional analysis method \\ 2.1 Capacity}

The calculation method of capacity at signalized intersection given in HCM2000 is based on the concept of saturation flow rate. When this method is applied, it is necessary to calculate capacity for each lane and each entrance. Its formula is shown as Eq. (1).

$$
c_{i}=s_{i} \frac{g_{i}}{C}
$$

Where

$c_{i}$ - Capacity of lane $i / \mathrm{veh} \cdot \mathrm{h}^{-1}$,

$s_{i}$ - Saturation flow rate for lane $i / \mathrm{veh} \cdot \mathrm{h}^{-1}$,

$g_{i} / C-$ Effective green ratio for lane $i$.

\subsection{Delay}

The calculation method of average control delay per vehicle (d) given in HCM2000 also needs to calculate for each lane one by one. This delay consists of uniform control delay $\left(d_{1}\right)$, random additional delay $\left(d_{2}\right)$ and initial queue additional delay $\left(d_{3}\right)$, as Eq. (2) shows. The specific formulas of the three kinds of delay mentioned above can be acquired in reference.

$$
d=d_{1}+d_{2}+d_{3}
$$

\subsection{Applicability analysis}

Based on analysis of Eq. (1), the capacity of certain lane is not only related to the signal control parameters, but also determined by the saturation flow rate in the lane. According to the analysis of delay calculation method mentioned in HCM2000 (2000), it can be found that, although the calculation formulas of three kinds of delay do not include the saturation flow rate parameter directly, include two main parameters those are the degree of saturation $X_{i}$ and capacity of lane $c_{i}$. However, the two important parameters are all determined by the saturation flow rate. From the analysis above, when capacity and delay in signalized intersection are calculated, the saturation flow rate of every lane is the key parameter.

According to HCM2000, the saturation flow rate of lane should be corrected based on the basic saturation flow rate of lane $\left(1900 / \mathrm{pc} \cdot \mathrm{h}^{-1} \cdot \mathrm{ln}^{-1}\right)$, considering several modifying factors, such as lane width, percentage of heavy vehicles, left or right turning lane and pedestrian crossing and so on. However, traffic operation characteristics of bus lane and traffic conflict are not considered as the modifying factors of saturation flow rate.

Further analysis showed that, because road-side bus lane is set at intersection entrance, the traffic conflicts between some buses and right-turn vehicles may occur at entrance. Therefore, the saturation flow rate of right-turn lane can not reach the calculated value of HCM2000. In addition, because of the bottleneck effect of bus stops through the road, the saturation flow rate of bus lane should also be reduced. In the other words, the saturation flow rate in the bottleneck of bus stop should be considered as the saturation flow rate of the whole bus lane. In view of the analysis above, it is necessary to consider the features of road-side bus lane for improvement of the calculation method of traditional saturation flow rate. This will contribute to analysis and calculation of capacity and delay in intersection entrance under such conditions.

\section{Investigation and data 3.1 Traffic survey}

Xinan Rd. and Wuyi Rd. signalized intersection in Dalian is selected as the investigation site in this research. As one of the most important traffic nodes in Dalian, the traffic load on this investigation is heavy. There are 7 bus lines in the south entrance of Xinan Rd. and 5 bus lines in the north entrance of Xinan Rd.. In order to realize the priority strategy of public transport, the two-way road-side bus lanes are set along the Xinan Rd. with the type of traffic marking. Therefore, the geometry and traffic condition of this intersection is appropriate for this research.

The traffic organization mode of this bus lane is intermittent bus travelling. In other words, it is a bus lane during the traffic 
peak hour at morning or afternoon. However, it also allows private vehicles travelling during the traffic off-peak hours. Therefore, the time interval when the lane acts as bus lane is selected as investigation time interval. Those are 7:00-9:00 and 16:00-18:00 every day. The investigation conducts 3 days from Tuesday to Thursday in one week. The traffic operating state at south and north entrance of Xinan Rd. during the investigation time interval can fully reflect the basic feature of road-side bus lane.

This investigation was divided into two stages, external observation and internal arrangement. In external observation, by mean of video observation, the traffic operating states at south and north entrance of Xinan Rd. are recorded. In internal arrangement, according to the video record, the traffic data can be acquired by means of artificial statistics.

\subsection{Situation of the intersection}

The intersection of Xinan Rd. and Wuyi Rd. is a X type fourway signalized intersection. The Xinan $\mathrm{Rd}$. is from south to north with two-way road-side bus lane, and the Wuyi Rd. is from west to east correspondingly. In order to avoid too many traffic conflicts between right-turn vehicle and buses in the scope of intersection, the bus lane is terminated at upstream entrance, a measurable distance away from the stop line. The lane layout, channelization mode and the setting type of bus lane of this intersection are shown as Fig. 1.

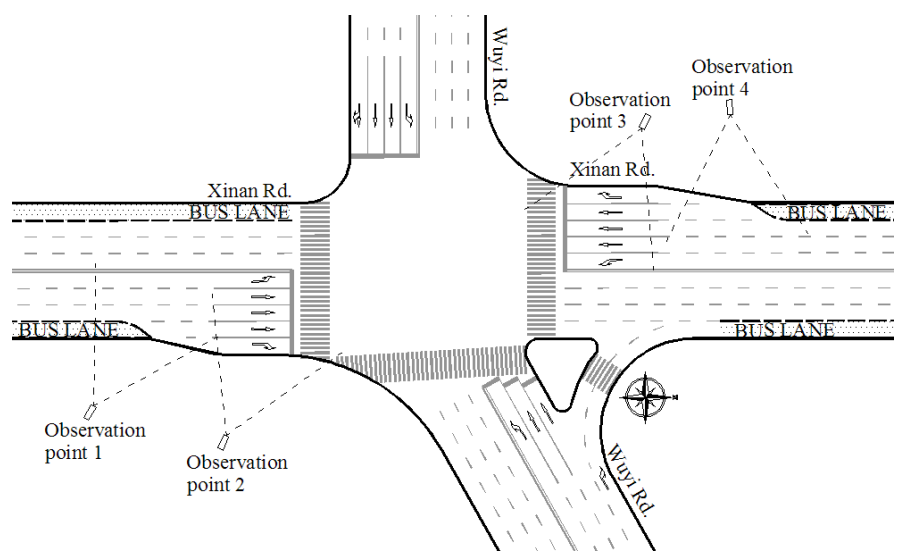

Fig. 1 Geometric construction of the intersection

\subsection{Traffic data acquisition}

After internal data arranging according to the video record, several kinds of traffic data of the two entrances can be acquired, such as the traffic volume of every lane and vehicle type, the headway of right-turn vehicles and buses, the queue length of every phase, the type and quantity of traffic conflicts and signal timing parameters. The data mentioned above will be used in determination of improved model's parameters and comparing analysis and calculation of the case. The signal cycle length of this intersection is $158 \mathrm{~s}$. The signal timing scheme is shown as Fig. 2.

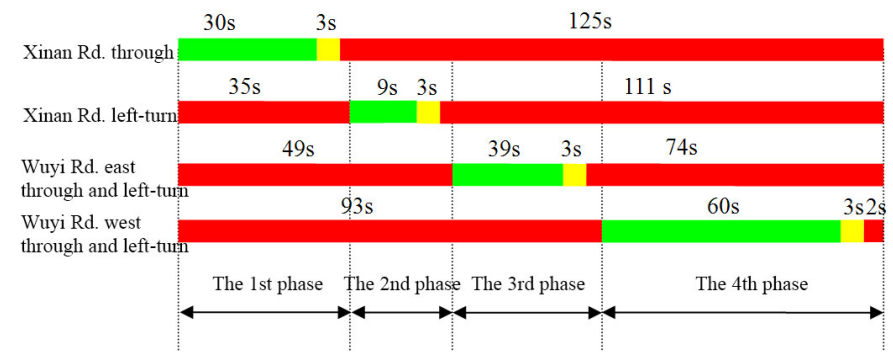

Fig. 2 The signal timing scheme of the intersection

Taking one signal cycle as an example, the traffic volume data at the south and north entrance of Xinan Rd. are listed in Table 1.

Table 1 The traffic volume in one signal cycle

\begin{tabular}{lcccccc}
\hline Entrance & \multicolumn{3}{c}{$\begin{array}{l}\text { North entrance } \\
\text { of Xinan Rd. }\end{array}$} & \multicolumn{2}{l}{$\begin{array}{l}\text { South entrance } \\
\text { of Xinan Rd. }\end{array}$} \\
\hline Direction & Through & Left-turn Right-turn & Through & Left-turn Right-turn \\
\hline Small & 45 & 1 & 60 & 39 & 2 & 41 \\
Middle & 2 & 1 & 4 & 3 & 0 & 2 \\
Large & 3 & 0 & 2 & 3 & 0 & 2 \\
Bus & 4 & 3 & 0 & 0 & 3 & 0 \\
\hline
\end{tabular}

\section{The saturation flow rate}

\subsection{Conflicts between right-turn and buses}

Because road-side bus lane is set in entrance and exclusive right-turn lane is set outside, traffic conflicts between a part of right-turn vehicles and buses will occur. According to the traffic investigation data, selecting the morning peak hour from 7:30 to $8: 30$ on the first day as analysis time interval, both the traffic conflicts and travelling delay caused by conflicts are analyzed. In this one hour, 47 times traffic conflicts between right-turn vehicles and buses occur. According to the travelling delay caused by conflicts, several groups are divided, as Table 2 shows. Based on the data in Table 2, the distribution of delay caused by conflict can be analyzed, as Fig. 3 shows.

Table 2 The data of traffic conflict and delay

\begin{tabular}{llll}
\hline Delay interval /s & $\begin{array}{l}\text { Conflicts } \\
\text { /times }\end{array}$ & Frequency & $\begin{array}{l}\text { Probability of } \\
\text { normal distribution }\end{array}$ \\
\hline $1.7-2.0$ & 2 & 0.043 & 0.064 \\
$2.0-2.3$ & 4 & 0.085 & 0.186 \\
$2.3-2.6$ & 8 & 0.170 & 0.401 \\
$2.6-2.9$ & 11 & 0.234 & 0.635 \\
$2.9-3.2$ & 9 & 0.191 & 0.738 \\
$3.2-3.5$ & 6 & 0.128 & 0.631 \\
$3.5-3.8$ & 5 & 0.106 & 0.396 \\
$3.8-4.1$ & 1 & 0.021 & 0.182 \\
$4.1-4.4$ & 1 & 0.021 & 0.062 \\
\hline
\end{tabular}




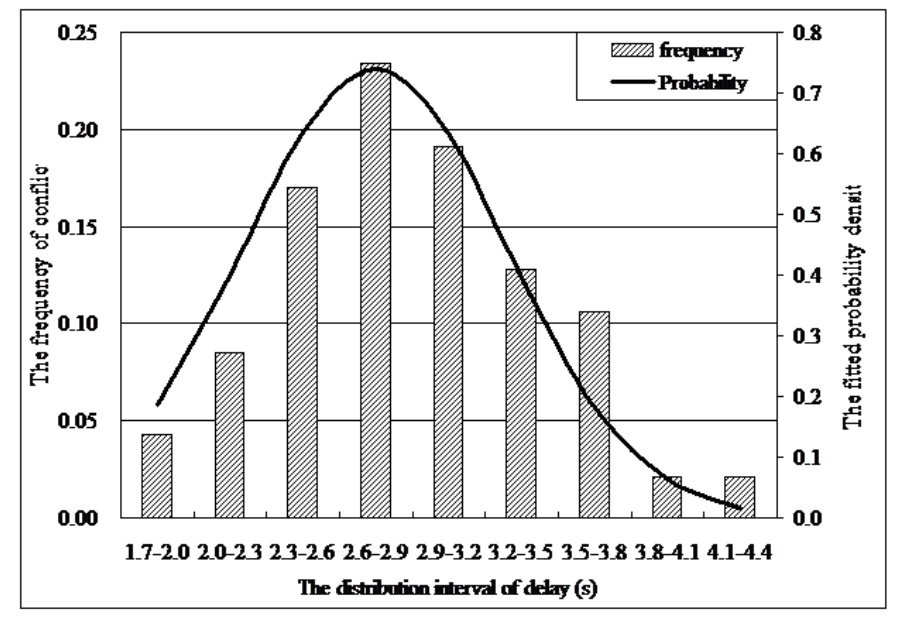

Fig. 3 The delay distribution of traffic conflict

From the data analysis above, the traffic conflicts between right-turn vehicles and buses excite indeed. They also affect travelling delay in different extant. The histogram means the frequency of traffic conflicts. The black line is the fitted probability density function (PDF) curve of traffic delay with the mean of $2.9 \mathrm{~s}$ and variance of 0.54 . Thus, the travelling delay caused by traffic conflict obeys the normal distribution approximately. In view of this kind of traffic conflict, the theory saturation flow rate of right-turn lane should be reduced or different probability function need to be used as concluded by Torok and Zefreh (2016).

\subsection{The saturation flow rate of right-turn lane}

The saturation flow rate of right-turn lane should be studied considering the arrangement and traffic operating feature of bus lane and right-turn lane. Two situations including static traffic and dynamic traffic should be discriminated for further discussion, according to the traffic status on the entrance of right-turn lane.

\section{(1) Static traffic}

This kind of traffic status means the vehicles in the bus lane are completely static at the entrance with exclusive right-turn lane. Normally, this kind of status appears when the queue length of vehicles in bus lane is longer than the length of exclusive right-turn lane, as shown in Fig. 4(a).

For this situation, right-turn vehicles can not travel through the bus lane and enter the right-turn lane. Therefore, in this time interval, the saturation flow rate of exclusive right-turn lane should be 0 . The starting moment of this time interval is when the vehicles in bus lane stop and plug the entrance of exclusive right-turn lane. The ending moment is when these vehicles start to move. This time interval can be marked as $t_{s}$.

\section{(2) Dynamic traffic}

This kind of traffic status means the vehicles in the bus lane are travelling at the entrance of exclusive right-turn lane, as Fig. 4(b) shows.

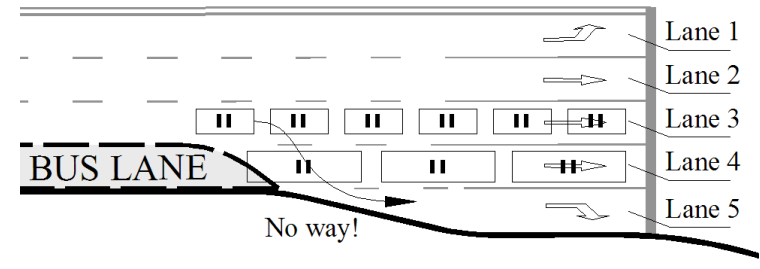

(a) The static traffic status

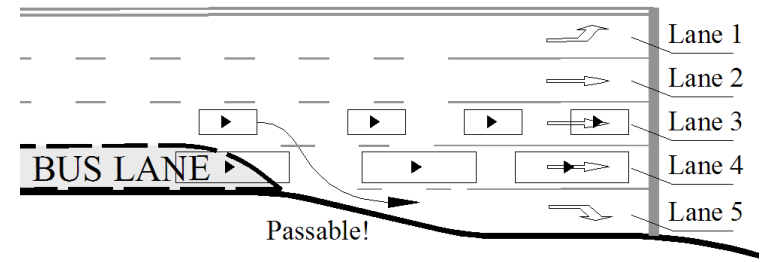

(b) The dynamic traffic status

Fig. 4 Two situations at the entrance of exclusive right-turn lane

For this situation, right-turn vehicles can realize right-turn travelling using acceptance gaps of the fleet in bus lane. If the signal cycle length is recorded as $C$, the duration of this status is $C-t$. Based on the Gap Acceptance Theory, the saturation flow rate of right-turn lane during this duration can be calculated by Eq. (3).

$$
s_{R i}=q_{B i} \frac{e^{-\lambda t_{0}}}{1-e^{-\lambda t}}
$$

Where

$S_{R i}$ - Saturation flow rate of right-turn lane when the bus fleet is travelling at entrance of right-turn lane in the $i$ th signal cycle $/ \mathrm{veh}^{-\mathrm{h}^{-1}}$,

$q_{B i}$ - Bus volume when the bus fleet is travelling at entrance of right-turn lane in the $i$ th signal cycle $/ \mathrm{veh} \cdot \mathrm{h}^{-1}$,

$t_{0}$ - Critical time interval of bus fleet/s, it means the minimum headway of bus for right-turn vehicle travelling through the bus fleet,

$t$ - Car-following headway of right-turn fleet for successive travelling through bus fleet/s,

$\lambda$ - Average arrival rate of buses in bus lane $/ \mathrm{veh} \cdot \mathrm{s}^{-1}$. It also equals to $q_{B i} / 3600$ s.

Synthesized the two kinds of situations mentioned above, the saturation flow rate of right-turn lane should be calculated using Eq. (4).

$$
s_{R}=\frac{1}{3600 T} \sum_{i=1}^{n} s_{R i}\left(C_{i}-t_{s i}\right)
$$

Where

$s_{R}-$ Average saturation flow rate of right-turn lane during the period of analysis $/ \mathrm{veh} \cdot \mathrm{h}^{-1}$,

$T$ - Length of analysis period $/ \mathrm{h}$,

$n$ - Number of cycle during analysis period,

$C_{i}$ - Signal cycle length of the $i$ th cycle/s,

$t_{s i}$ - Length of time when bus fleet stops at entrance of rightturn lane in the $i$ th cycle/s. 


\subsection{The saturation flow rate of bus lane}

The determination method of saturation flow rate mentioned in HCM2000 published by Transportation Research Board (2000) indicates that, if there is a bus stop in range of intersection, the adjustment factor $f_{b b}$ for blocking effect of local buses should be considered. Therefore, the basic saturation flow rate should be corrected caused by the bottleneck effect of bus stop, according to stopping characteristic at the upstream stopping of bus lane. Then, the value after adjusted can be used as the saturation flow rate of bus lane.

\subsection{The saturation flow rate of the medial lane adjacent to bus lane}

Analyzing the traffic operation state shown in Fig. 4(b), it can be found that, when right-turn vehicles in the medial adjacent lane of bus lane arrive at the entrance of right-turn lane, if there is a passable headway of bus fleet in bus lane, the rightturn vehicles can realize right-turn travelling smoothly. However, if the bus fleet does not satisfy the condition of travelling through, these right-turn vehicles must travel very slowly of stop in order to wait for the passable gap appearing. This situation will lead delay of subsequent vehicles in the adjacent lane. Thus, setting of bus lane will also affect the saturation flow rate of its medial adjacent lane.

For these reasons, referring the analysis method of the effect of bus stop on subsequent vehicles travelling mentioned in HCM2000, the saturation flow rate of the medial lane adjacent to bus lane can be derived. It can be acquired after a correction, based on saturation flow rate of conventional lane, as Eq. (5) shows.

$$
s_{a}^{\prime}=s_{a}\left(1-\frac{k \cdot t_{c}}{3600}\right)
$$

\section{Where}

$s_{a}^{\prime}-$ saturation flow rate of the medial lane adjacent to bus lane after correction $/ \mathrm{veh} \cdot \mathrm{h}^{-1}$,

$s_{a}$ - saturation flow rate of conventional lane calculated according to HCM2000/veh $\cdot h^{-1}$,

$k$ - quantity of delayed right-turn vehicles blocked by bus fleet/veh,

$t_{c}-$ average delay pre vehicle blocked by bus fleet/s.

Synthesizing the analysis above, it should be recognized that, because of setting road-side bus lane, all of the saturation flow rates of right-turn lane, bus lane and the medial lane adjacent to bus lane vary. Therefore, the saturation flow rate parameters after corrected should be used to analyze the capacity and delay of signalized intersections.

\section{Application study}

\subsection{Saturation flow rate calculation of every lane}

Using the data acquired from the traffic investigation, saturation flow rates of every lane in north and south entrance of the Xinan Rd. and Wuyi Rd. intersection can be calculated. Each lane is numbered from inside to outside, as Fig. 4(a) and (b) shows. Among them, the saturation flow rates of right-turn lane, bus lane and the medial lane adjacent to bus lane are all calculated by both HCM2000 method and the improved method in this research. However, the saturation flow rates of other lanes are still calculated by HCM2000 method.

The adjustment factors for lane width of every lane are all 0.983 , which is converted by HCM standard. The adjustment factors for heavy vehicles can be acquired by the average value of the traffic investigation in three days. The adjustment factor for left turns and right turns should be 0.95 and 0.85 correspondingly according to HCM. When the improved method is applied, the quantities of right-turn vehicles blocked by bus $k$, the average delays $t_{c}$, the adjustment factors for bus stopping $f_{b b}$ and bus volume $q_{B i}$ can be acquired by calculating the average value of actual investigation data. The critical time intervals of bus fleet $t_{0}$ and the car-following headway of rightturn fleet $t$ are $4.5 \mathrm{~s}$ and $2.5 \mathrm{~s}$ correspondingly according to the recommended values given by the research of Shi-ming Wang (2010). The calculated saturation flow rates of every lane in north and south entrance of Xinan Rd. are listed in Table 3.

Table 3 The calculation results of saturation flow rate

\begin{tabular}{|c|c|c|c|c|}
\hline \multirow{2}{*}{$\begin{array}{l}\text { Saturation } \\
\text { flow rates } \\
/ \mathrm{pcu} \cdot \mathrm{h}^{-1}\end{array}$} & \multicolumn{2}{|c|}{$\begin{array}{l}\text { North entrance } \\
\text { of Xinan Rd. }\end{array}$} & \multicolumn{2}{|c|}{$\begin{array}{l}\text { South entrance } \\
\text { of Xinan Rd. }\end{array}$} \\
\hline & HCM2000 & $\begin{array}{l}\text { Improved } \\
\text { model }\end{array}$ & HCM 2000 & $\begin{array}{l}\text { Improved } \\
\text { model }\end{array}$ \\
\hline Lane 1 & 1365 & & 1721 & \\
\hline Lane 2 & 1693 & & 1707 & \\
\hline Lane 3 & 1693 & 1542 & 1707 & 1587 \\
\hline Lane 4 & 1528 & 1170 & 1507 & 1095 \\
\hline Lane 5 & 1527 & 1366 & 1526 & 1354 \\
\hline
\end{tabular}

From the Table 3, it can be found that, from lane 3 to lane 5, the calculation results of the improved model are all less than that of HCM. Among them, the reduction of the medial lane adjacent to bus lane (Lane 3 ) is the least one. This indicates the effect of traffic conflict on this lane is less. For bus lane (Lane 4), the reduction of saturation flow rate is larger, after considering the bottleneck effect of bus stop. The saturation flow rate of right-turn lane (Lane 5) is also less than that without considering the probability of travelling through. In addition, the saturation flow rates of left-turn lane in the south and north entrance are very different from each other. The reason for this situation is that the heavy vehicle's percentage of leftturn vehicles in north entrance is much higher than that of south 
entrance. This leads the adjustment factor for heavy vehicles is much less than that of south entrance.

\subsection{Capacity calculation of every lane}

According to the calculation method of capacity given in HCM2000, capacity of every lane can be calculated, by multiplying the saturation flow rate of every lane and the green ratio for the corresponding lane. In this intersection, all of the rightturn vehicles are not restricted by traffic signal. The saturation flow rate of the right-turn exclusive lane can be determined as its capacity directly. The calculated capacities of every lane are listed in Table 4.

Table 4 The calculation results of capacity of every lane

\begin{tabular}{lllll}
\hline Capacity & \multicolumn{2}{c}{$\begin{array}{c}\text { North entrance } \\
\text { of Xinan Rd. }\end{array}$} & \multicolumn{2}{c}{$\begin{array}{c}\text { South entrance } \\
\text { of Xinan Rd. }\end{array}$} \\
\cline { 2 - 5 } pcu $\mathrm{h}^{-1}$ & HCM2000 & $\begin{array}{l}\text { Improved } \\
\text { model }\end{array}$ & HCM2000 & $\begin{array}{l}\text { Improved } \\
\text { model }\end{array}$ \\
\hline Lane 1 & 86 & 86 & 109 & 109 \\
Lane 2 & 332 & 332 & 335 & 335 \\
Lane 3 & 332 & 303 & 335 & 311 \\
Lane 4 & 300 & 230 & 296 & 215 \\
Lane 5 & 1527 & 1366 & 1526 & 1354 \\
\hline
\end{tabular}

\subsection{Average delay calculation of every lane}

Taking the traffic flow parameters of north entrance in Xinan Rd. investigated in $15 \mathrm{~min}$ as an example, the average delays pre vehicle of every lane can be calculated. The green ratio is converted according to the actual signal timing scheme. The saturation degree $x$ is the quotient of actual measured traffic volume divided by the capacities of lane which is calculated by HCM2000 and the improved model respectively. The uniform control delay $d_{1}$, random additional delay $d_{2}$ and initial queue additional delay $d_{3}$ are all calculated by means of HCM. The parameters and several kinds of the delay are listed in Table 5.

According to the data of actual investigation and by means of parameter determination above, the average delays pre vehicle of every lane in north and south entrance of Xinan Rd. can be calculated and shown in Fig. 5.

It can be seen from the histogram in Fig. 5 that, the traffic characteristics of north and south of Xinan Rd. are similar to each other. Because the green time of the left-turn phase is shorter, the capacity of left-turn lane (Lane 1) is lower, the average delay of left-turn vehicles is larger. Because of the larger traffic volume of the through private lane (Lane 2 and Lane 3), its saturation degree is higher, the average delay of through private vehicles is much larger. For the bus lane (Lane 4), there are several private vehicles travelling in it illegally, so delay of vehicles still exists. Because the right-turn vehicles in the right-turn lane travel without the restriction of traffic signal, the average delay is extremely low. Further analysis indicated that, because of setting bus lane, it is necessary for the rightturn vehicles in the medial lane adjacent to bus lane to travelling through the bus fleet, in order to right turning.

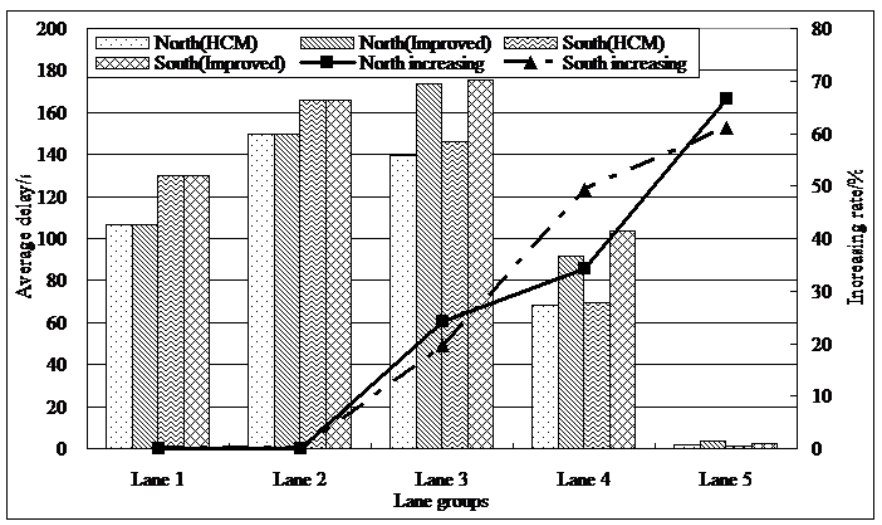

Fig. 5 The average delay of every lane in the north and south entrances of Xinan Rd.

The dashed and continuous lines in Figure 5 show the increasing rate of delay calculated by paper's method comparaed with the traditional (HCM) method. Because the Lane 1 and 2 are the inside lane. They are not affected by right-turn vehicles. Thus the delays of them are no difference between the two methods. The Lane 3, 4 and 5 are the medial lane adjacent to bus lane, bus lane and right-turn lane respectively. The average delays are all larger than the results calculated by traditional (HCM) means, becaused of the effect of traffic conflicts between rightturn vehicles and buses. It can also be recognized that the effect of this conflict on delay of right-turn vehicles is larger than that of the medial lane adjacent to bus lane. After considering the bottleneck effect at upstream bus stop of bus lane, the average delay calculated by the improved method is also larger than

Table 5 The average delay of Xinan Rd. north entrance

\begin{tabular}{|c|c|c|c|c|c|c|c|c|c|c|c|c|}
\hline \multirow{2}{*}{$\begin{array}{l}\text { Methods } \\
\text { Parameters }\end{array}$} & \multicolumn{6}{|c|}{ HCM 2000} & \multicolumn{6}{|c|}{ The improved model } \\
\hline & $\lambda$ & $x$ & $d_{l} / \mathrm{s}$ & $d_{2} / \mathrm{s}$ & $d_{3} / \mathrm{s}$ & $d / \mathrm{s}$ & $\lambda$ & $x$ & $d_{l} / \mathrm{s}$ & $d_{2} / \mathrm{s}$ & $d_{3} / \mathrm{s}$ & $d / \mathrm{s}$ \\
\hline Lane 1 & 0.06 & 0.77 & 72.86 & 33.49 & 0.00 & 106.34 & 0.06 & 0.77 & 72.86 & 33.49 & 0.00 & 106.34 \\
\hline Lane 2 & 0.20 & 1.08 & 64.83 & 73.80 & 10.84 & 149.48 & 0.20 & 1.08 & 64.83 & 73.80 & 10.84 & 149.48 \\
\hline Lane 3 & 0.20 & 1.02 & 63.78 & 54.07 & 21.69 & 139.54 & 0.20 & 1.12 & 65.34 & 84.27 & 23.76 & 173.38 \\
\hline Lane 4 & 0.20 & 0.73 & 59.53 & 8.92 & 0.00 & 68.45 & 0.20 & 0.95 & 62.70 & 29.17 & 0.00 & 91.87 \\
\hline Lane 5 & 1.00 & 0.75 & 0.00 & 2.05 & 0.00 & 2.05 & 1.00 & 0.83 & 0.00 & 3.41 & 0.00 & 3.41 \\
\hline
\end{tabular}


that calculated by traditional method in HCM. However, this effect is the medium of the two mentioned above.

\section{Conclusions}

(1) Road-side bus lane set at entrance of signalized intersection will lead right-turn vehicles in its medial adjacent lane conflict with bus fleet. This traffic conflict causes the capacities of right-turn lane and the medial lane adjacent to bus lane to reduce. It also causes the average delay to increase.

(2) Considering the bottleneck effect of upstream bus stop in bus lane, the capacity of bus lane is reduced to a much greater extent. The average delay of buses increases correspondingly.

(3) This research is based on the traffic investigation of two entrances at a typical signalized intersection. Therefore, there is a certain particularity in terms of investigation data and influence analysis. However, the general influence acquired by this study still can be referred by the similar researches.

\section{Acknowledgments}

This research was supported by the Research Fund for the Doctoral Program of Liaoning Province under the granted number 20141109 and the Dalian Support Project of Youth Star of Science and Technology under the granted number 2016RQ055. We also acknowledge the editors and anonymous reviewers, for their detailed suggestions, precise comments and continuous helps, which lead to the belief that their dedication contributes to this research and it would not have been feasible without their support.

\section{References}

Arasan, V. T., Vedagiri, P. (2010) Study of the impact of exclusive bus lane under highly heterogeneous traffic condition. Public Transport. 2(1), pp. 135-155. https://doi.org/10.1007/s12469-010-0021-x

Chen, X., Yu, L., Jia, X., Gong, H. (2014) Analytical Procedures for Estimating the Capacity of the Urban Expressway Section near Off-ramps and On-ramps with a Median Exclusive Bus Lane. Journal of Transportation Engineering. 140(3), pp. 1-9. https://doi.org/10.1061/(ASCE)TE.1943-5436.0000642

Chiabaut, N., Xie, X., Leclercq, L. (2012) Road Capacity and Travel Times with Bus Lanes and Intermittent Priority Activation. Transportation Research Record. 2315, pp. 182-190. https://doi.org/10.3141/2315-19

Gu, T., Wu, S. (2013) Analysis of Bus Lane Effect on Arrival Intervals of Buses. Journal of Shijiazhuang Railway Institute. 26(1), pp. 97-100.

Hu, S. S., Wang, Z. W. (2012) Research on the Bus-stop Traffic Capacity Model for Bus Lanes. Journal of Transport Science and Engineering. 28(1), pp. 79-83.
Ismail, R., Hafed, M. H., Ismail, A. (2012) Implementing Specific Lane Length on Bus Route for Operation Delays Reduction. Journal of Applied Sciences. 12(19), pp. 2077-20819. https://doi.org/10.3923/jas.2012.2077.2081

Lei, L. G. (2008) Impact of Bus Lane on Road Traffic. Beijing Jiaotong University, pp. 35-47.

Lu, B., Niu, H. M. (2011) Setting Condition and Effect Analysis of Bus Lanes at Signalized Intersection. Journal of Lanzhou Jiaotong University. 30(6), pp. 127-131.

Surprenant-Legault, J., El-Geneidy, A. (2011) Introduction of Reserved Bus Lane: Impact on Bus Running Time and On-time Performance. Transportation Research Record. (2218), pp. 10-18. https://doi.org/10.3141/2218-02

Transportation Research Board (2000) Highway Capacity Manual. Chapter 16. pp- 9-26. Transportation Research Board, Washington, D.C. 2000.

Török, A., Zefreh, M. M. (2016) Assessing the Need for Applying Multimodal Speed Distribution in Road Transport Macro Emission Estimation. Acta Technica Jaurinensis. 9(2), pp. 118-127. https://doi.org/10.14513/actatechjaur.v9.n2.402

Wang, S.M., Xu, J.M. (2010) Analysis of Gap Acceptance Control Strategies and Traffic Parameters. Science Technology and Engineering. 10(7), pp. 1804-1807.

Wang, T., Chen, J. (2014) Traffic Volume Conditions of Setting Bus Lane on Urban Roadway Based on Time Utility. Journal of Harbin Institute of technology. 46(4), pp. 115-121.

Zhao, J., Yang, X. G., Bai, Y., Lao, Y. P. (2008) Investigation on Impacts of Bus Lane on Capacities of Signalized Intersections. Urban Transport of China. 6(5), pp. 74-79. https://doi.org/10.13813/j.cn11-5141/u.2008.05.008

Zhu, C. J. (2006). Delay Research in Signalized Intersection with Bus Lane. Harbin Institute of technology, pp. 16-55. 